\title{
FUENTES-RODRÍGUEZ, C. Y ÁLVAREZ-BENITO, G. (2016): A GENDER- BASED APPROACH TO PARLIAMENTARY DISCOURSE. AMSTERDAM / PHILADELPHIA: JOHN BENJAMINS. 230 PÁGINAS
}

\author{
MARINA JimÉNEZ RODRÍGUEZ \\ Universidad de Sevilla \\ marina_jr12@hotmail.com
}

Este trabajo aborda el estudio del discurso político desde una perspectiva de género. En concreto, analiza los mecanismos lingüísticos y las estrategias discursivas para lograr la persuasión en las intervenciones políticas y las diferencias de estos mecanismos y estrategias en hombres y mujeres. Se trata de un corpus de gran utilidad para aquellos investigadores que analicen el discurso político desde una perspectiva global, ya que lleva a cabo una descripción muy completa del discurso parlamentario y de los elementos lingüísticos que lo componen, como la ritualización de los discursos ideológicos, los procesos de intensificación, la comunicación no verbal o el uso de la emoción, entre otros.

Tras una amplia introducción en la que se presentan aspectos como el tema, la conexión con los estudios previos sobre el discurso parlamentario, los objetivos de la investigación o su estructura, comienza el desarrollo de cada uno de los nueve capítulos presentes en el libro, cada uno de ellos referido a un mecanismo persuasivo del discurso político y su relación con la variable género.

En el capítulo 1, Women in the Andalusian Parliament (La mujer en el Parlamento andaluz), de Fuentes Rodríguez, encontramos una contextualización documentada acerca del papel de la mujer en el Parlamento andaluz, el primero en España en contar con paridad de diputados, de ahí su elección para este libro, como explican las editoras en la parte introductoria. Fuentes Rodríguez plantea en este apartado cómo la presencia de la mujer ha ido creciendo de forma constante, hasta llegar a hoy, donde nos encontramos con el mismo reparto de escaños entre hombres y mujeres. Se trata de un fenómeno reciente que ha tenido como resultado el salto del $5,5 \%$ de presencia de mujeres en la primera legislatura al $47,7 \%$ en la novena (Rodríguez, 2011).

Este trabajo aborda un estudio documental acerca del peso de la mujer en el ámbito parlamentario andaluz, atendiendo a factores como el interés en la política, la predisposición a

Para citar esta reseña / To cite this book review: Jiménez Rodríguez, Marina (2017). Reseña de Catalina Fuentes-Rodríguez y Gloria Álvarez-Benito (2016): A gender-based approach to parliamentary discourse. Amsterdam / Philadelphia: John Benjamins. (230 páginas). ELUA, 31: 387-392. doi:10.14198/ ELUA2017.31.23

Enlace / Link: http://dx.doi.org/10.14198/ELUA2017.31.23

Este trabajo se publica bajo una licencia de Creative Commons Reconocimiento-NoComercial 4.0 Internacional 
la confrontación o la colaboración o el grado de participación. Se relata, asimismo, la evolución de la paridad en el Parlamento a lo largo de las legislaturas. Como nos señala la autora, la mayor presencia de la mujer en el Parlamento no siempre va acompañada de una mayor participación, que resulta menor, de forma especial en el caso de las sesiones plenarias.

En el capítulo 2, Intensification, identity and gender in the Andalusian Parliament (Intensificación, identidad y género en el Parlamento Andaluz), Fuentes Rodríguez reflexiona sobre una de las estrategias argumentativas más frecuentes en el discurso político, la intensificación. En concreto, estudia el marcador discursivo es que desde una perspectiva multidimensional. A través del análisis de diferentes fragmentos de las sesiones de la octava legislatura, Fuentes Rodríguez plantea las funciones discursivas de la enfatización en el ámbito del DP. Una de las aportaciones esenciales de este estudio es que realiza un análisis cuantitativo para determinar las relaciones entre intensificación y roles interactivos: si se recurre más a esta estrategia por parte de miembros del gobierno o de la oposición, si depende del rol institucional o de la ideología y, lo que resulta el hilo conductor de todos los artículos de este volumen, si el factor género tiene relevancia en el uso de la intensificación como estrategia argumentativa.

Fuentes Rodríguez concluye a través de este capítulo cómo la intensificación, además de para expresar la opinión y generar la imagen del hablante como una persona hábil desde el punto de vista comunicativo, es un rasgo característico del discurso político, esencial para configurar la identidad como diputados. En referencia a la variable género, la aportación de este artículo es de gran relevancia, ya que demuestra que el género no tiene influencia en el uso de la intensificación. Este hecho es especialmente pertinente porque contradice estudios previos que señalaban que el discurso femenino se caracteriza por la cortesía verbal y la mitigación (Lakoff, 1973; 1975). Como demuestra este capítulo, la intensificación en mujeres diputadas no reproduce patrones de inseguridad ni denota subestimación propia.

En el capítulo 3 del volumen, Gender differences in enumerative series (Diferencias de género en las series enumerativas), Brenes Peña continúa reflexionando sobre los procesos de intensificación, dando lugar a un valioso estudio sobre las series enumerativas. $\mathrm{Su}$ principal planteamiento es, además de identificar los aspectos formales que caracterizan la fomulación de este recurso, determinar si existe una diferencia en el uso de las series enumerativas en función de la variable género. Las series enumerativas, eficaces para el enaltecimiento de las tareas realizadas por el candidato o su endogrupo, así como para el ataque al exogrupo, son uno de los recursos de intensificación más frecuentes en el DP. A través del análisis de 40 preguntas orales, que la autora estudia tanto desde una perspectiva cualitativa como cuantitativa, Brenes Peña lleva a cabo un estudio pionero en su ámbito, ya que hasta el momento de la publicación de este volumen no existían estudios que abordaran las series enumerativas en función de las diferencias de su uso entre hombres y mujeres.

En las 109 series enumerativas que este trabajo ha estudiado, la autora plantea que esta estrategia, que aporta énfasis y fuerza a las intervenciones, puede encontrarse bajo tres formas distintas: la sinonimia, la enumeración por relación de proximidad o por relación de gradualidad. A través de este capítulo, Brenes Peña extrae las siguientes conclusiones: que su uso es más frecuente en la segunda intervención de los diputados, que la relación entre los elementos enumerados es de adición, así como que se trata de una estrategia que responde a la perfección a la naturaleza interactiva de la pregunta oral, con un efecto social descortés. Esta 
conclusión va en la línea de lo que ya afirmaba Fuentes Rodríguez (2012), cuando se refería a la pregunta oral como uno de los géneros para dañar la imagen de la oposición (2012: 133).

Finalmente, en cuanto al tema principal de este volumen, las diferencias de género, contrariamente a lo planteado en el capítulo 2, en el caso de las series enumerativas sí existe una predominancia en el uso por parte de hombres. Sin embargo, cabe señalar aquí que las diferencias son mínimas, por lo que la autora alerta de la necesidad de abordar este estudio desde otros géneros discursivos antes de extraer conclusiones significativas al respecto.

En el capítulo 4 de este libro, Argumentation and face-threatening acts. The non-literal quotation (Argumentación y actos que amenazan la imagen. La cita no literal), López Martín aborda un análisis de la parodia como recurso de descalificación del adversario político a través de la cita no literal. Además, plantea una profundización de las estrategias falaces y agresivas relacionadas con el género, tanto del hablante como del interlocutor, lo que constituye una de las principales aportaciones de este capítulo a la investigación sobre argumentación. Para este análisis se han tenido en cuenta 56 preguntas orales, 28 de hombres y 28 de mujeres. La cita no literal, un mecanismo que crea polifonía en el discurso, es un medio altamente eficaz de manipulación y altamente rentable desde el punto de vista comunicativo, que le permite al diputado demostrar su ingenio y ganarse la aprobación de dos sectores: su propio partido y el electorado. La parodia puede ir dirigida a adversarios concretos, al partido político enemigo en su conjunto o aludiendo a la comunidad o al sentido común, llevando a cabo lo que se conoce como populist fallacy.

Este capítulo, innovador en su planteamiento a la hora de relacionar parodia y género, muestra que, al igual que veíamos con las series enumerativas, se trata de un recurso más empleado por hombres. Sin embargo, la explicación que López Martín aporta aquí rechaza que se deba al factor género. Como apunta el autor, lo que verdaderamente resulta determinante en el uso de la parodia es la posición de poder, ocupada de forma mayoritaria por hombres. Esto es, para López Martín, la causa de que exista un mayor uso por parte de diputados masculinos.

En el capítulo 5 del volumen, Pseudo-desemantisation as a discursive strategy in political discourse (Pseudo-desemantización como estrategia discursiva en el discurso político), García Platero y Castillo Carballo analizan el fenómeno lingüístico de la desemantización como estrategia discursiva en el ámbito del discurso político. Mientras en los capítulos anteriores se abordaban análisis cuantitativos de los distintos usos en función del factor género, en este artículo no se ha trabajado con datos, sino que se ha profundizado en el funcionamiento de la neutralización semántica, empleada por los diputados para atenuar los aspectos negativos al tiempo que se intensifican los positivos.

En este trabajo, García Platero y Castillo Carballo se centran en la colocación léxica como estrategia de intensificación argumentativa a través de las llamadas unidades pleonásticas. En concreto, analizan el cliché léxico hacer un esfuerzo, del que concluyen que tiene una doble significación: cuando el político la emplea para justificar decisiones difíciles ante la ciudadanía y cuando se informa de un esfuerzo que tienen que realizar los ciudadanos, en cuyo caso pierde su significado original y lo sustituye por un valor de imposición. Otro de los fenómenos estudiados en este capítulo es la pseudo-desemantización a través de colocaciones pleonásticas, como es el caso de decepcionante y demagogia. Aunque no se observan diferencias de género en el uso de la desemantización, este trabajo permite observar cómo las selecciones léxicas se corresponden a un proceso estratégico acorde a funciones ideológicas, que actúan como un mecanismo de intensificación argumentativa. 
En el capítulo 6, Lexical colloquialisation in commissions of the Andalusian Parliament (Coloquialización léxica en las comisiones del Parlamento Andaluz), González Sanz analiza desde un prisma cualitativo y cuantitativo la coloquialización como estrategia en el discurso político, basándose en 134 intervenciones parlamentarias de un total de cuatro comisiones. Además de presentar una descripción del español coloquial y del proceso de coloquialización (Clayman y Heritage, 2002: 339), González Sanz se adentra en la dinámica parlamentaria y el nivel de espontaneidad de cada una de las fases. En este capítulo encontramos un análisis cuantitativo de los siguientes elementos: palabras sin significado específico (hacer, eso, cosa y tema), que se encuentran presentes en el $35 \%$ de los discursos parlamentarios; empleo de léxico coloquial -montón, pillar, churro, tufo o tocho- y vulgarismos (cachondeo). Por último, otro de los elementos que González Sanz tiene en cuenta para este análisis es el estudio de las unidades fraseológicas, como los proverbios, las colocaciones o las frases hechas.

La principal novedad que incorpora este capítulo es el estudio riguroso y completo de los usos coloquiales en el lenguaje parlamentario español. Además, en la línea temática de este volumen, se incorpora una comparativa de género, de la que se extrae que las diferencias en el uso de estructuras coloquiales entre hombres y mujeres no son significativas. En el caso de palabras sin significado específico, encontramos 1,52 unidades por intervención en hombres, frente a las 0,80 en el caso de las mujeres. En lo que se refiere al empleo de léxico coloquial, en hombres se da un 0,11 de unidades por intervención, frente a los 0,4 en el caso de mujeres. Las unidades fraseológicas son el rasgo coloquial en el que menos diferencias existen en función del género, con 0,56 unidades por intervención en hombres y 0,35 en mujeres. Esto se explica, según la autora, por tratarse del rasgo coloquial más típico en el ámbito del discurso parlamentario.

El capítulo 7, Emotional argumentation in political discourse (Argumentación emocional en el discurso político), lleva a cabo un análisis sobre otro de los elementos del discurso político, la emoción. Alcaide Lara, Carranza y Fuentes Rodríguez analizan en este trabajo la emoción como valor argumentativo en el ámbito parlamentario. Este estudio resulta especialmente interesante, ya que aporta varias novedades en un campo en el que se ha trabajado poco, la emoción desde un prisma discursivo. Estudia los elementos mediante los cuales se formula la emoción en el discurso político, abriendo la puerta a futuras investigaciones de índole cuantitativa. Este trabajo es indispensable para aquellos investigadores que deseen abordar un análisis cuantitativo sobre el uso de la emoción en el discurso político, ya que establece una clasificación de elementos discursivos que le sirven al político para provocar emociones: elementos léxicos, marcadores discursivos, intensificación, expresiones del tipo "nuestros hijos", metáforas, preguntas retóricas, cambios de registro o expresiones rituales.

Este trabajo, basado en la Teoría de la evaluación de Martin (2000) y Bednarek (2006), así como en la Teoría de la modalidad (Bally, 1944) y de la argumentación (Anscombre y Ducrot, 1983; Lo Cascio, 1998) pone de manifiesto tanto los objetivos del uso de la emoción en el discurso político, así como sus múltiples ventajas argumentativas, como el aumento de la veracidad, el acercamiento del discurso al lenguaje cotidiano, la desritualización de la argumentación, así como la capacidad de la emoción como un argumento en sí mismo.

El capítulo 9 de este volumen, Gender differences in eye-contact behaviour in parliamentary discourse (Diferencias de género en el comportamiento de contacto visual en el discurso parlamentario), aborda la identificación de las funciones que cumple el contacto visual en el ámbito político, así como una comparativa en función de las variables turno de palabra, partido y género. Las funciones que Álvarez-Benito e Íñigo-Mora señalan tras el análisis son las si- 
guientes: permite que el discurso suene más natural y disminuye la formalidad de la lectura de la pregunta oral; regula la conversación y el desarrollo de los turnos de palabra; revela afiliación y lealtad hacia el máximo representante de la formación; expresa desacuerdo; actúa como un elemento reforzador de la argumentación y refleja las relaciones de poder, entre otras funciones.

El análisis de doce preguntas orales -un total de 48 de turnos de palabra- revela que la mayoría de los contactos visuales se presentan al final del segundo turno de palabra, donde el discurso es más espontáneo. En el primer turno, el contacto visual cumple una función reguladora, mientras que el segundo actúa para expresar desacuerdo y como refuerzo argumentativo. En cuanto a las diferencias en función del partido al que corresponden los diputados, Álvarez-Benito e Íñigo-Mora concluyen que este recurso de comunicación no verbal tiene una mayor presencia en los diputados socialistas. En cuanto al análisis de esta temática en función del género, el hilo conductor de este volumen, las autoras concluyen que, al contrario que lo que sostenían autores como Rubin (1970) o Harper et al. (1994), son los hombres los que hacen más uso de este recurso no verbal.

El último de los capítulos de este volumen es Time, gender and parliamentary discourse (Tiempo, género y discurso parlamentario) y en él Bañón Hernández, Arcos Urrutia y Requeña Romero analizan la importancia de la gestión de los tiempos y su relación con el factor género. Partiendo de la hipótesis de que aspectos como las pausas, la duración y la velocidad de los discursos contienen un valor argumentativo, estos autores analizan en este trabajo distintas interacciones de los consejeros andaluces Antonio Ávila y Mar Moreno. Con el objetivo de explicar la relevancia del tiempo en los discursos, estos autores se detienen en el estudio de los siguientes parámetros: duración en segundos, número de sílabas, de sílabas por segundo, de palabras, de palabras por segundo; número de pausas, duración y media de estas, así como su porcentaje en relación con el total de la duración total de las intervenciones.

En cuanto a las aportaciones en materia de diferencias de género, este trabajo permite al investigador conocer que la pausa interdiscursiva en el caso masculino es más de un segundo mayor que la diputada Moreno (0,421 frente a 0.311), así como que las pausas son más cortas cuando la interacción es con miembros del propio partido, ya que la función es, en estos casos, de intensificación. En el caso de la diputada Moreno, este trabajo permite conocer que la gestión del tiempo en sus intervenciones varía en función del género del interlocutor: la pausa interdiscursiva está por debajo de la media cuando se dirige a mujeres, mientras que se encuentra por encima de la media cuando se dirige a hombres. En cuanto al consenso y al diálogo, estos autores concluyen que es más valorado en el caso de las mujeres que en el de los diputados hombres.

En conclusión, este volumen constituye un estudio imprescindible para la investigación en el ámbito del discurso político, así como para desterrar determinadas consideraciones existentes acerca de las diferencias de género en el lenguaje político. Su principal aportación al estudio del discurso parlamentario es que se trata de una investigación sobre diferencias de género basada en un corpus. Además, la institución estudiada, el Parlamento andaluz, es especialmente interesante como objeto de estudio, ya que cuenta con equilibrio de mujeres y hombres desde la octava legislatura, siendo la primera cámara española en conseguir dicho reparto de escaños. Este factor es, como ha demostrado este trabajo, un elemento fundamental en la inexistencia de diferencias de género en el discurso parlamentario.

La principal pregunta que se planteaba este volumen al inicio, ¿las mujeres, incorporadas con posterioridad al Parlamento hacen uso de diferentes estrategias que los hombres?, ha sido resuelta con gran claridad a lo largo de los diferentes artículos que componen el volu- 
men. Esta investigación ha permitido demostrar que las mujeres han adoptado las mismas estrategias y mecanismos lingüísticos que empleaban los hombres, no porque consideren estos rasgos como masculinos, sino porque han sido identificados como rasgos de discurso profesional y, como tales, han sido asumidos y legitimados mediante su uso. Además, ha quedado patente que el género no es un factor de importancia en el discurso político, sino que lo que es un elemento verdaderamente determinante a la hora de generar diferencias discursivas entre los miembros parlamentarios es el rol: si el diputado pertenece al gobierno o a la oposición; el partido del que forme parte, así como su rol en la Cámara.

Constituye, asimismo, el primer volumen que analiza las estrategias y mecanismos que emplean tanto hombres como mujeres a la hora de alcanzar sus fines políticos. Se centra en la intensificación, la emoción y la coloquialización. Precisamente la primera de estas estrategias constituye otro de los ámbitos en los que se ha podido demostrar que algunas consideraciones previas sobre género y discurso no coinciden con la realidad, al menos en el caso del Parlamento andaluz. Hasta esta investigación se sostenía que las mujeres empleaban más la atenuación que los hombres (Lakoff, 1973; 1975). Sin embargo, este ambicioso volumen ha demostrado que emplean este recurso de igual manera, por lo que no existen diferencias de género relevantes en el ámbito del discurso parlamentario.

Se trata, pues, de una recopilación esencial para investigaciones que aborden el análisis del discurso político, tanto para aquellos trabajos que empleen una perspectiva de género como para aquellos que analicen otras áreas del DP sin entrar en diferencias entre hombres y mujeres, ya que también se identifican aquí los principales mecanismos argumentativos empleados. En definitiva, un elaborado estudio cualitativo y cuantitativo que resuelve los interrogantes sobre el modo de hablar de las mujeres en la política y que es una guía fundamental para cualquier estudio futuro sobre el discurso parlamentario.

\section{Referencias bibliográficas}

Anscombre, J. C. and O. Ducrot (1983). L'argumentation dans la langue. Lieja: Pierre Mardaga.

Bally, C. (1965[1944]) Linguistique générale et linguistique française. Berna: Francke, 4th ed.

Bednarek, M (2006). Evaluation and cognition. Inscribing, evoking and provoking opinión. En H. Piswa (ed.) Language and Memory. Aspects of Knowledge Representation. Berlin: 187-221.

Clayman, S. and J. Heritage (2002). The News Interview: Journalists and Public Figures on the Air. Cambridge: Cambridge University Press. doi: 10.1017/CBO9780511613623

Fuentes-Rodríguez, C. (2012). Las preguntas orales en el Parlamento español. Philologia Hispalensis 26/1-2: 129-150.

Harper et al. (1994). Sexual harassment and immediacy behaviors in the multicultural workplace: a communication paradox. in Michael B. Goodman (ed.) Corporate Communication:Theory and Practice. Albany, New York: State University of New York Press.

Lakoff, R. (1973). Language and woman's place, in Language in Society 2 (1):45-79. doi: 10.1017/ S0047404500000051

Lakoff, R. (1975). Language and woman's place. New York: Harper and Row Publishers.

Lo Cascio, V. (1998). Gramática de la argumentación. Madrid: Alianza.

Martin, J. R. (2000). Beyond Exchange: APPRAISAL Systems in English in S. Hunston and G. Thompson (eds.) Evaluation in Text. Oxford: Oxford University Press, 142-175.

Rodríguez, R. (2011). Diputadas. La mujer en el Parlamento de Andalucía y en la política local, autonómica y nacional. Sevilla: Publicaciones Parlamento de Andalucía.

Rubin, Z. (1970). Measurement of romantic love in Journal of Personality and Social Psychology, 16: 265-273. doi: $10.1037 / \mathrm{h} 0029841$ 